\title{
Health Literacy: More Than a One-Way Street
}

\author{
Author: Karama C. Neal ${ }^{\text {a }}$ \\ Affiliation: a Emory University, \\ DOI: 10.1080/15265160701638652 \\ Publication Frequency: 12 issues per year \\ Published in: The American Journal of Bioethics, \\ Volume 7, Issue 11 November 2007 , pages 29 - 30 \\ First Published on: 01 November 2007
}

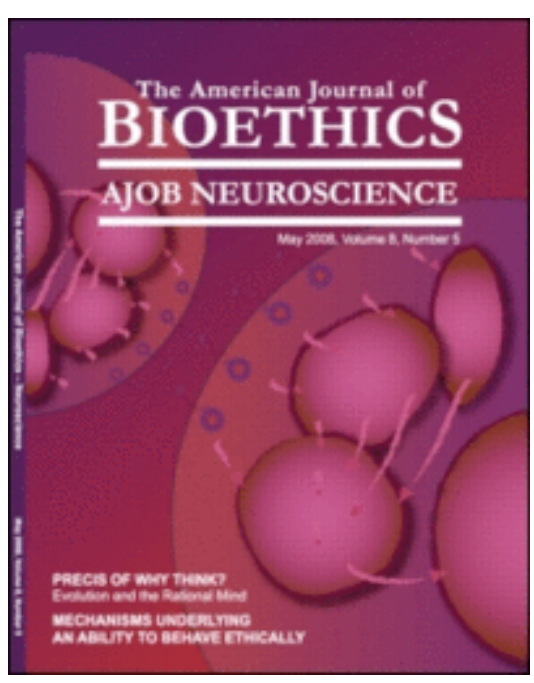

Know how to listen, and you will profit even from those who talk badly.-Plutarch

The following are the universally fundamental laws of literary communication: 1 . One must have something to communicate; 2 . One must have someone to whom to communicate it; 3 . One must really communicate it, not merely express it for oneself alone. Otherwise it would be more to the point to remain silent.-Friedrich Von Schlegel

I commend Drs. Volandes and Paasche-Orlow (2007) on their effort to focus our collective energy on justice and health literacy in the United States healthcare system. Linguistic literacy can be defined as "an individual's ability to read, write, speak in English, compute and solve problems at levels of proficiency necessary to function on the job, in the family of the individual and in society" (Workforce Investment Act of 1998, n.p.). This definition is useful because it includes the ability to both deliver and receive information in a way that is mutually understood. By analogy, one can define health literacy to include the ability to both transmit and comprehend health-related information. This process requires at least two individuals who are literate in the medium of communication. However, much of the work on health literacy focuses primarily on the patient's ability to comprehend and use health-related information. Here, I will discuss how the inclusion of multiple stakeholders in the definition of health literacy leads to additional recommendations for improving our healthcare system and making it more just.

The skills required for health literacy and health communication are critical not only for patients, their family members and caregivers, but also for their healthcare providers. The common definition of health literacy and the one used by Volandes and Paasche-Orlow (2007) is "the degree to which individuals have the capacity to obtain, process and understand basic health information and services needed to make appropriate health decisions" (5). The definition is not limited to those whose health is under consideration. Everyone involved in the delivery and receipt of healthcare is included in this definition. Healthcare providers, social workers, hospital finance officers, and others regularly "obtain, process and [ideally] understand" $(2007,5)$ health-related information not only from their colleagues, but, importantly, from their patients, and often from caregivers and family members as well.

It is critical that patients, providers, and others involved in the healthcare system learn and exhibit the ability to speak with clarity and listen with understanding. If we excuse any stakeholders from one or more of these responsibilities, we not only endanger health outcomes (as Volandes and Paasche-Orlow [2007] outline), but 
Health Literacy: More Than a One-Way Street - The American Journ... http://www.informaworld.com/smpp/section?content=a785910101\&f...

we also send a clear if implicit message about whose voices are most important. While it is critical that providers explain medical information and patients understand it, if physicians are primarily taught to speak clearly and patients are only taught to listen carefully, then there is a significant amount of useful information that will be left uncommunicated. Patients, and often their families and caregivers, have valuable knowledge to share with healthcare providers about their health goals, their symptoms, and their personal and family health history, for example. Physicians must be able to "obtain, process and understand" (Volandes and

Paasche-Orlow 2007, 5) this information to identify healthcare options and make appropriate care recommendations to their patients (Sachs Hills 2006). Likewise, patients should be encouraged to share health-related information that could be useful in providing for their care.

Volandes and Paasche-Orlow (2007) propose several ways to help patients navigate the healthcare system and understand and use complex and sometimes frightening medical information. Additional recommendations can be made by considering the ability of healthcare providers to listen to and learn from their patients. For example, during healthcare discussions, providers should pause periodically to confirm that they fully understand the patient. This is in addition to pausing to confirm that the patient understands them. In as much as pay-for-performance plans undermine the informed consent process, physicians and healthcare providers must be especially vigilant about acknowledging patients' questions, concerns, and decisions about proposed tests and treatments.

Language differences can create additional communication difficulties. Increased language training and use of interpreters are an important part of the solution (Yeo 2004). These skills are important since it can be burdensome to ask family members, often children, to serve as translators when both receiving and conveying complex and sometimes frightening medical information (Free et al. 2003). Inaccuracies in translation can also have a negative impact on health outcomes (Free et al. 2003). Use of family members as translators may also create privacy concerns.

Similarly, cultural competency education can facilitate communication in the healthcare arena even when there is a shared language. A provider's ability to understand traditional medical practices, religious beliefs, or use of alternative medical terminology can be crucial in delivering appropriate care to the patient. It is useful to define 'culture' to include groups that are not simply defined by ancestral ethnicity (e.g., Barnett 1999; Interpreter of Maladies 2005). Cultural competency education, as with all activities, is most effective when the ethical foundation is also considered (Paasche-Orlow 2004).

Volandes and Paasche-Orlow (2007) do us all a service by directing our attention to health literacy and suggesting we orient the health-care system around those people who are least well-off. Such a system necessitates egalitarian communication with information transmission and understanding in at least two directions. Even a simplified version of our healthcare system will require multidirectional communication between many stakeholders. Participation by all parties in all aspects of health literacy and health communication will result in a more just healthcare system and better health outcomes for all segments of the population.

\section{REFERENCES}

1. Barnett, S. (1999) Clinical and cultural issues in caring for deaf people. Family Medicine 31:1, pp. 17-22. [ pubmed ] [ csa ]

2. Free, C., Green, J., Bhavnani, V. and Newman, A. (2003) Bilingual young people's experiences interpreting in primary care: A qualitative study. British Journal of General Practice 53 , pp. 530-535. [ csa ]

3. Interpreter of Maladies (2005) Interpreter of maladies: Will "cultural competency" reduce care disparities?. Applied Neurology - http://www.appneurology.com/showArticle.jhtml ?articleld=175400220 December 1. Available at (accessed August 25, 2007) 
Health Literacy: More Than a One-Way Street - The American Journ... http://www.informaworld.com/smpp/section?content=a785910101\&f...

4. Paasche-Orlow, M. (2004) The ethics of cultural competence. Academic Medicine 79 , pp. 347-350. [ csa ] [ crossref ]

5. Hills, L. Sachs (2006) Becoming a better listener. Journal of Medical Practice Management 21:6, pp. 348-350. [ csa ]

6. Shilling, L. M. and Fuller, L. K. (1997) Dictionary of quotations in communications Greenwood Press , Westport, CT

7. Volandes, A. E. and Paasche-Orlow, M. K. (2007) Health literacy, health inequality and a just healthcare system. American Journal of Bioethics 7:11, pp. 5-11.

8. http://www.doleta.gov/regs/statutes/wialaw.txt Workforce Investment Act of 1998. Public Law 105-220 112 Stat. 936. Available online at (accessed August 25, 2007)

9. Yeo, S. (2004) Language barriers and access to care. Annual Review of Nursing Research 22 , pp. 59-73. [ csa ] [ pubmed ] 